\title{
JUNO Jubilee: Bringing Real-Life Experiences to the Classroom!*
}

\author{
Teresa R. Taggart, MS, CNMT \\ Program Director, Nuclear Medicine Technology, Cuyahoga Community College, Parma, Ohio
}

\begin{abstract}
As Program Director of the only Nuclear Medicine Technology program in Northeast Ohio, I am always looking for ways to simulate real life to the students before they enter the clinical experience at the local hospitals. Through the community bond initiative, our classroom was granted funds to acquire the Human Patient Simulator (HPS) JUNO, supplied through CAE Health Care, which was delivered late 2018.
\end{abstract}

J Nucl Med Technol 2019; 47:215-216

DOI: 10.2967/jnmt.119.232991

UNO is a highly functional manikin. It comes with programmable clinical experiences simulating several common patient ailments and audible complaints accompanying them. The JUNO simulator has multiple preprogrammed opportunities to allow for basic nursing processes such as suctioning of the lungs, endotracheal tube placement, subclavian catheter placement, urinary catheter and ostomy stoma care and maintenance, and allows administration of intravenous fluid and medication. Although the software is designed to serve many modalities with training, there are several advantages that serve nuclear medicine specifically quite nicely. We can have audible respiratory and heart sounds, have accessible antecubital and dorsal veins to start intravenous infusion, simulate CPR bagging for ventilation purposes, have various case study analyses and the vital signs of that type of patient, have a simulated cardiac arrest complete with a "My chest is hurting" verbal warning with signals going off to suggest electrocardiogram changes, and have vital sign monitoring complete with palpation of venous blood vessels for pulse rate detection.

The most advantageous aspect of JUNO for our curriculum is the ability for JUNO to be programmable with individualized case studies. These case analyses provide the students a patient background of other disciplinary testing and results and current evaluation of the patient as well as

Received Jun. 26, 2019; revision accepted Aug. 1, 2019.

For correspondence or reprints contact: Teresa R Taggart, Cuyahoga Community College, 11000 Pleasant Valley Rd., Health Careers A221b, Parma, OH 44130.

Email: teresa.taggart@tri-c.edu

*Portions of this article first appeared in the June/July 2019 issue of ASRT Scanner and are reprinted with permission.

COPYRIGHT @ 2019 by the Society of Nuclear Medicine and Molecular Imaging. allow for investigation into how to care for the "patient" once we have them in our department. The modules have respiratory, laboratory, cardiac, and pathology values for each scenario.

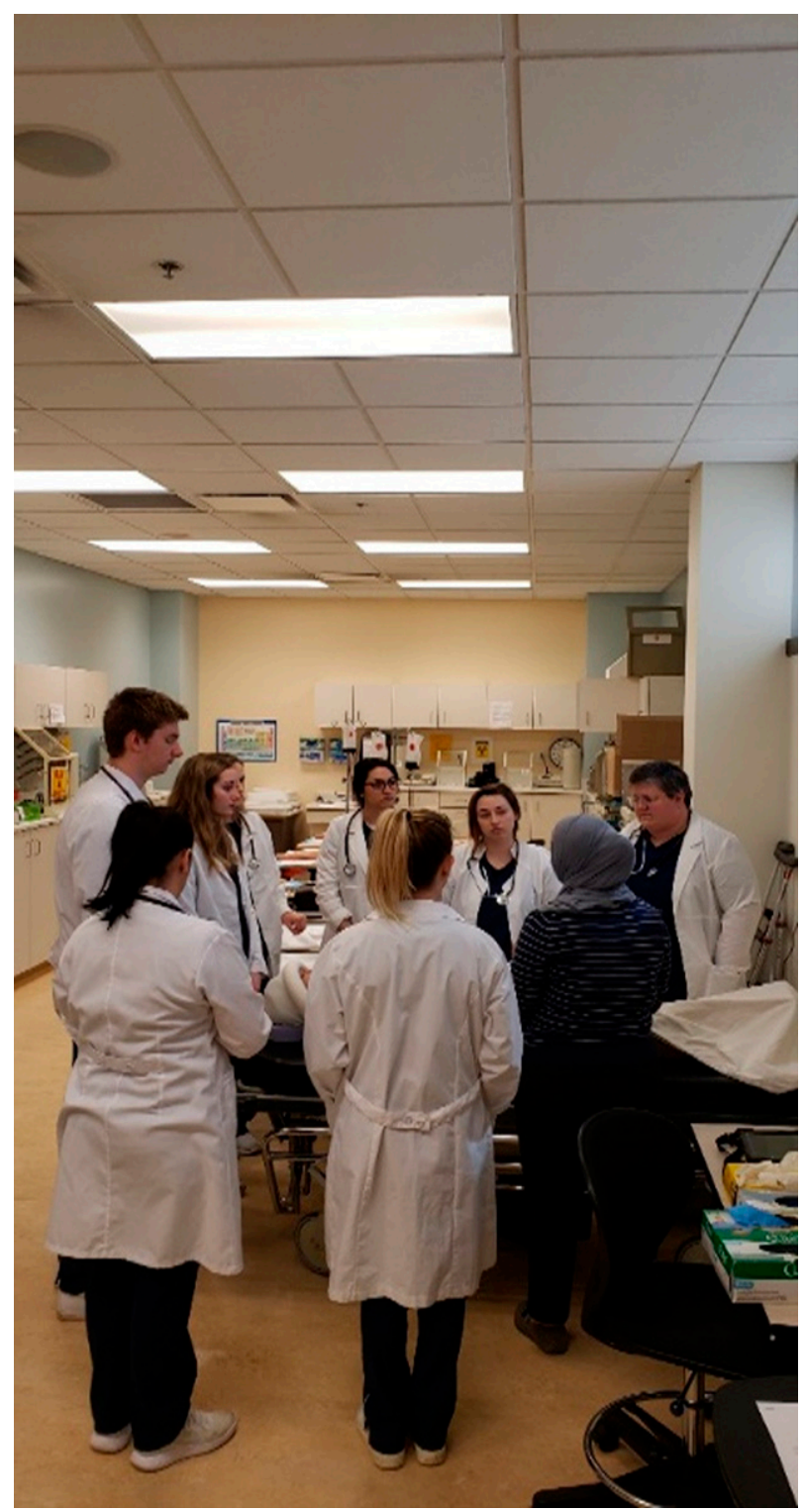

FIGURE 1. Hands-on learning. 
A nuclear medicine educator has individualized a cardiac myocardial infarction into modules as well as the gastrointestinal bleed and hepatobiliary dysfunction studies and uses the dedicated computer to teach electrocardiogram patterns most commonly seen by nuclear medicine technologists. By having case studies presented, the students can progress from patient history and intake to describing basic nuclear medicine protocol and imaging techniques, to analyzing the correlated laboratory and other modality results, and come up with an anticipated diagnosis. This helps introduce the interprofessional team management concept to the classroom and shows the students how diagnostic imaging is interrelated and an integral part of patient care.

The realistic skin of JUNO provides an excellent canvas for the students to attach and remove electrocardiogram lead patches and connect the 12 lead cables to learn proper placement for superior electrocardiogram tracings. Although JUNO weighs approximately 50 pounds, it is possible to use the HPS in patient skills classes, teaching movement and transfer techniques in order to provide the safest patient care when on the hospital floors and in real life.

For our classroom, it is very useful to simulate a code situation, such as when the students are practicing in one area and they hear JUNO cry out "Ow, that hurts." Or maybe they hear "My chest hurts! I feel funny." Alarms are going off, signaling electrocardiogram abnormalities or vital signs out of range of normal, and it is essential that the students assess the situation. They decide to call a code if necessary, simulate intravenous insertion, start bagging the patient, gather vital signs, scribe the incident, and continue until a "Doctor" arrives and shuts off the alarms, signaling the end of the nuclear medicine department's involvement. Through years of experience, the code situation provides a student more angst than any other task within the department, so our goal is to provide training time within the classroom so that they can anticipate and react quickly in the field.

As educators, our hopes are that all students' professional legacies will have a strong foundation due to what we provide for them now. While "Miss Juno" has been used by only one cohort of students so far, we anticipate that it will complement the classroom training tremendously. If we can cement the theory of high patient quality of care early into the students' experience through simulation and case scenarios, we can expect this behavior to be carried into their professional lives when they leave for clinical experiences.

Currently, this cohort of students has completed one semester of clinical experience and both students and clinical site personnel all say that this class appears to be a more comfortable group than in the past. I would like to believe it is because of their exposure and preparation within the classroom and with "Miss Juno" that set their apprehension to rest before arriving at the hospital sites.

$\mathrm{CAE}$ provided personalized training, where we had 4 instructors receive about $3 \mathrm{~h}$ of training. This was invaluable, as we became the trainers of others when necessary. CAE is a wonderful company to work with, providing numerous opportunities and rapid responses to our inexperienced questions. Their warranties are of highest regard for our investment and they understand our dedication to our student experiences. 